\title{
The Impact of an Integrated Gratitude Intervention on Positive Affect and Coping Resources
}

\author{
Jeffrey Klibert ${ }^{1}$ (D) Haresh Rochani ${ }^{1}$. \\ Hani Samawi ${ }^{1}$ - Kayla Leleux-LaBarge ${ }^{1}$. \\ Rebecca Ryan ${ }^{1}$
}

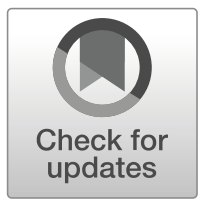

Accepted: 16 April 2019 / Published online: 27 April 2019

(C) Springer Nature Switzerland AG 2019

\begin{abstract}
Gratitude interventions are an emerging focus in clinical work. Consistent with theories of positive emotions, research needs to examine the effects of gratitude on different wellbeing outcomes after a positive experience. To this end, the current study examines the effects of a gratitude intervention on positive emotions and coping resources (resilience and coping self-efficacy) after a positive experience. Forty-eight university students completed a positive experience exercise and were then randomly assigned to either a gratitude or control task. Results revealed a significant interaction effect, such that individuals in the gratitude intervention group reported higher levels of positive emotions (at time 3) compared to the control group. This finding supported the hypothesis that gratitude maintains positive emotions resulting from a positive experience. Our findings also revealed a significant main effect of condition on resilience and coping self-efficacy. Specifically, the gratitude intervention group reported higher levels of resilience and coping self-efficacy compared to the control group. These findings support the hypothesis that gratitude interventions employed after a positive experience impact coping resources. The results are further discussed in terms of gratitude being an effective upregulation strategy.
\end{abstract}

Keywords Gratitude · Positive emotion regulation · Positive emotions · Coping resources

Gratitude is an emotional state marked by active attempts to mindfully notice and appreciate positive aspects of life (Wood et al. 2010). Researchers characterize gratitude as a popular and promising focus of study given its strong connection to increased wellbeing and life satisfaction (Rash et al. 2011). For instance, experimental findings highlight gratitude as a key factor in reducing stress and depression ( $\mathrm{O}^{\prime}$ Leary and

Jeffrey Klibert

jklibert@georgiasouthern.edu

1 Georgia Southern University, 2670 Southern Drive, Statesboro, GA 30460, USA 
Dockray 2015), reinforcing social connectivity (Kerr et al. 2015), and boosting subjective reports of happiness (Seligman et al. 2005). However, much is left to investigate, in terms of clarifying pathways by which gratitude promotes psychological wellbeing (Davis et al. 2016).

Identifying mechanisms that upregulate positive emotions and build coping resources is a decisive step in helping therapists promote personal growth and emotional wellbeing in their clients (Seligman 2011; Wood and Tarrier 2010). Guided by different positive psychological theories (Fredrickson 2001; Gross 1998), the purpose of the current research was to experimentally determine if a gratitude intervention would (a) maintain positive emotions generated from a positive experience task and (b) promote higher levels of coping resources (resilience and coping self-efficacy).

\section{Gratitude as an Upregulation Strategy}

Research outlines the practical benefits of increasing positive emotions to produce more enduring wellbeing outcomes (Tugade et al. 2014). Specifically, positive emotions generate more flexible and inclusive thinking and problem solving strategies by (a) facilitating unique cognitive associations, (b) extending processes by which people develop and implement different cognitive resources, and (c) promoting greater levels of creativity and openness in perspective taking (Isen 1999). In turn, these broadened cognitive styles buffer against the negative effects of stress and prevent the onset of different psychopathological conditions (e.g., Gross and Muñoz 1995), while at the same time promote important wellbeing outcomes including purpose in life, social support, life satisfaction, and resilience (Cohn et al. 2009; Fredrickson et al. 2008).

Emotion regulation is an emerging field of study concerned with the processes by which individuals manage when and how they experience and express specific emotions (Gross 1998). Typical goals of emotional regulation include the downregulation of negative emotions (e.g., minimizing the experience of distressing feelings of fear, sadness, disgust, etc.) and the upregulation of positive emotions (e.g., maintaining and enhancing desired feelings of joy, enthusiasm, contentment, etc.; Gross 2015). Despite the concurrent importance of these two goals, there is a relative dearth of studies outlining the procedures and mechanisms by which individuals upregulate positive emotions (Quoidbach et al. 2015). However, the Process Model of Emotion Regulation (PMER; Gross 1998) offers a framework by which emotion regulation strategies can be implemented through different stages of the emotion-generative process. Specifically, Gross (1998) offers five sets of regulatory strategies designed to alter the emotional trajectory given a specific regulation goal. These strategies include: (1) situation selection - approaching and/or avoiding specific stimuli to increase the likelihood of experiencing a desired emotion, (2) situation modification - shaping/altering an environment to promote a desired emotion, (3) attention deployment - isolating focus on aspects of an experience that reinforce a desired emotion, (4) cognitive change transforming perceptions to alter an emotional trajectory in the desired direction, and (5) response modulation - altering physiological, experiential, or behavioral responses to an existing emotional expression.

Gratitude interventions are the most extensively examined cognitive change strategy (Quoidbach et al. 2015). Specifically, daily reflection on aspects of life for which one is 
grateful intensifies and prolongs the experience of several positive emotions including enthusiasm, attentiveness, and alertness (Emmons and McCullough 2003). Moreover, expressions of gratitude serve to suppress the fleeting nature of positive emotions by cognitively reframing the strength and utility of positive experiences and generating new pathways by which individuals benefit from positive experiences more completely through various psychological systems (e.g., cognitive, behavioral, physiological; Bryant 2003; Bryant and Veroff 2007).

Despite these assertions, support for gratitude as an effective emotional regulation strategy is mixed (Quoidbach et al. 2015). Some researchers found that practicing gratitude over an extended period of time predicts elevations in happiness and wellbeing scores compared to practicing neutral activities (Layous et al. 2013). In addition, individuals who engage in higher levels of gratitude (i.e., counting blessings) are more likely to report higher levels of overall happiness across time (Jose et al. 2012). However, several studies fail to garner support for group differences (gratitude intervention vs. control) on measures of positive emotions in student and clinical samples (Kerr et al. 2015; Sheldon and Lyubomirsky 2006), suggesting gratitude may not be well suited to upregulate positive emotions.

The lack of consistent findings is one reason why researchers call for more creative experimental designs to investigate the upregulation effects of gratitude on positive emotions (Wood et al. 2010). Most studies examine gratitude's influence on positive emotions outside the context of positive experiences. To maximize the effectiveness of different regulation strategies, clinicians and researchers need to consider the time frame in which gratitude interventions should be deployed within the emotiongenerative process (Quoidbach et al. 2015). Specifically, different strategies are better deployed at different times (i.e., before, during, or after) within the emotion generation process. For instance, it would be interesting to determine if cognitive change strategies, like gratitude interventions, are effective in terms of maintaining or enhancing positive emotions after a positive experience (Wood et al. 2010).

\section{Gratitude and Coping Resources}

Another pathway by which gratitude is thought to contribute to wellbeing is through resource development. Consistent with the broaden-and-build theory (Fredrickson 2001), gratitude is an adaptive evolutionary mechanism designed to broaden momentary thought-action repertoires marked by heightened appreciation for others and prosocial behavior (Fredrickson 2004). In turn, these broadening effects help individuals engage with their environment and goals in a manner that promotes new skill acquisition and lasting psychological resources. In the literature, researchers consistently find a causal link between gratitude expressions and social resource development. For instance, gratitude interventions play an active role in helping individuals mobilize existing support systems, forge new social relationships, and strengthen current social relationships (Kerr et al. 2015). However, much less is known about how gratitude contributes to the development of coping resources, like resilience and coping self-efficacy.

Although few studies offer direct evidence for a causal relationship between gratitude and coping resources, several studies highlight positive associations between these 
variables. Of note, individuals who express higher levels of gratitude report greater success in coping with stress and adversity (Wood et al. 2010). For instance, among high-risk samples, high gratitude individuals exhibit more post-traumatic growth, a concept closely aligned to resilience, in response to adversity (Ruini and Vescovelli 2013; Vernon et al. 2009). Gratitude traits are also associated with reduced risk to different psychopathological outcomes across time (Wood et al. 2008). Finally, individuals high in gratitude often engage in a number of behaviors known to promote resilience and coping self-efficacy. Specifically, gratitude expressions are correlated with positive re-appraisal, planning, and goal-directed strategies that reduce the frequency and intensity of stress and adversity (Wood et al. 2007). These correlational findings are supported by qualitative reports from adolescents and young adults recovering from cancer. Different gratitude themes (i.e., benefit-finding, meaning making) appear to be foundational in how adolescents and young adults develop and maintain resilient and self-efficacious perspectives in the face of overwhelming adversity (Rosenberg et al. 2014). In light of these findings, gratitude appears well-suited to increase coping resources (i.e., resilience, coping selfefficacy) outside of social support.

\section{Current Study}

Given the emergence of gratitude as an important tool in clinical work, there is a clear need to experimentally validate pathways by which gratitude contributes to wellbeing (Wood et al. 2010). Using experimental designs to test the effectiveness of gratitude interventions is a necessary area of expansion in the field of positive psychology. Within the field, gratitude interventions are assumed to upregulate positive emotions and build lasting coping resources (Davis et al. 2016). Research is needed to thoroughly validate these effects in the context of positive experience.

Drawing from the tenets of emotion regulation theories (Fredrickson 2004; Gross 2015), we examined the parameters by which a gratitude intervention contributes to different wellbeing outcomes. The goals of the current study are two-fold. First, we sought to determine if a gratitude intervention could upregulate positive emotions that resulted from a positive experience task. Second, we evaluated whether or not inducing gratitude would result in higher levels of resilience and coping self-efficacy. In light of applicable theory and empirical work, we hypothesized that individuals who participated in a gratitude intervention after a positive experience would (1) report higher levels of positive emotions and 2) report higher levels of resilience and coping selfefficacy compared to control group participants. By examining these lines of inquiry, we hope to provide an empirical foundation by which clinicians can employ gratitude interventions as a means to effectively improve human functioning.

\section{Method}

\subsection{Participants}

Participants were recruited through an online recruitment system and received course credit and/or extra credit for participating. The final sample included 48 students 
enrolled in a large southeastern university in the United States. The majority of the participants self-identified as women $(n=34,70.8 \%)$, while the remaining participants self-identified as men $(n=14,29.2 \%)$. Participants ranged in age between 18 and 23 with an average age of $19.25(S D=1.14)$. The participants identified as European American $(n=31,64.6 \%)$, African American $(n=13,27.1 \%)$, Mexican American/ Latino(a) $(n=1,2.1 \%)$, and multiracial/other $(n=3,6.3 \%)$.

\subsection{Procedure}

A flow chart of the study procedures can be found in Fig. 1. The beginning of the study entailed participants taking a brief online survey. The survey included demographic questions, a request for contact information, and a measure of wellbeing (see below). After completion of the survey, participants were asked if they would participate in the second component of the study. Of the initial 270 participants, 180 volunteered for the second component and those individuals were included in the Rank Set Sampling (RSS; McIntyre 1952) procedure to select the final sample of participants. Using simple random assignment with small sample sizes may not adequately disperse characteristics relevant to the treatment effects equally across all conditions (Kazdin 2016). This limitation is even more pronounced given the large number of constructs known to affect individual differences in the expression and regulation of emotions (Gross 1998, 2015). To combat these limitations, we employed a RSS scheme to ensure important characteristics (i.e., wellbeing) known to affect emotional regulation efforts are equally dispersed across all conditions.

We employed a RSS scheme based on variation in wellbeing score ranks, the auxiliary covariate obtained in the initial survey, to determine which participants were selected to participate from the original 180 volunteers. RSS is an alternative method to simple random sampling (SRS) in estimating the population mean. Research highlights RSS as a more powerful, unbiased, and efficient method of parameter estimation when compared against SRS (Samawi and Abu-Dayyeh 2002). In addition, consideration for auxiliary covariates in the RSS scheme can improve the precision by which treatment effects are detected (Donner and Zou 2007) and reduce the sample size needed to detect clinically significant treatment effects (Egger et al. 1985).

Participant selection was determined by the implemented RSS scheme. First, the 180 participants were randomly shuffled and divided into two groups with each group containing 90 participants. We then divided participants randomly into 10 subsets with 9 participants per subset. From the first two subsets (one from each group), we ranked the first 3 participants based on wellbeing scores and selected the participants with only minimum wellbeing scores to be included for participation. We randomly assigned participants to one of the two conditions (gratitude vs. control). Unselected students were discarded. We repeated the same step on the next 3 participants for each subset, but selected only participants with moderate wellbeing scores to be included for participation. Selected participants were randomly assigned to one of the two conditions and unselected participants were discarded. We repeated this step once more on the next 3 participants for each subset, but selected only participants with maximum wellbeing scores to be included for participation. Selected participants were randomly assigned to one of the two conditions and unselected participants were discarded. From these steps, we obtained two groups of three participants selected based on the ranking 
270 participants completed Initial survey; 180 agreed to volunteer for experiment

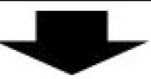

Rank Set Sampling Procedures using Well-Being as a covariate were implemented to select sample of 60

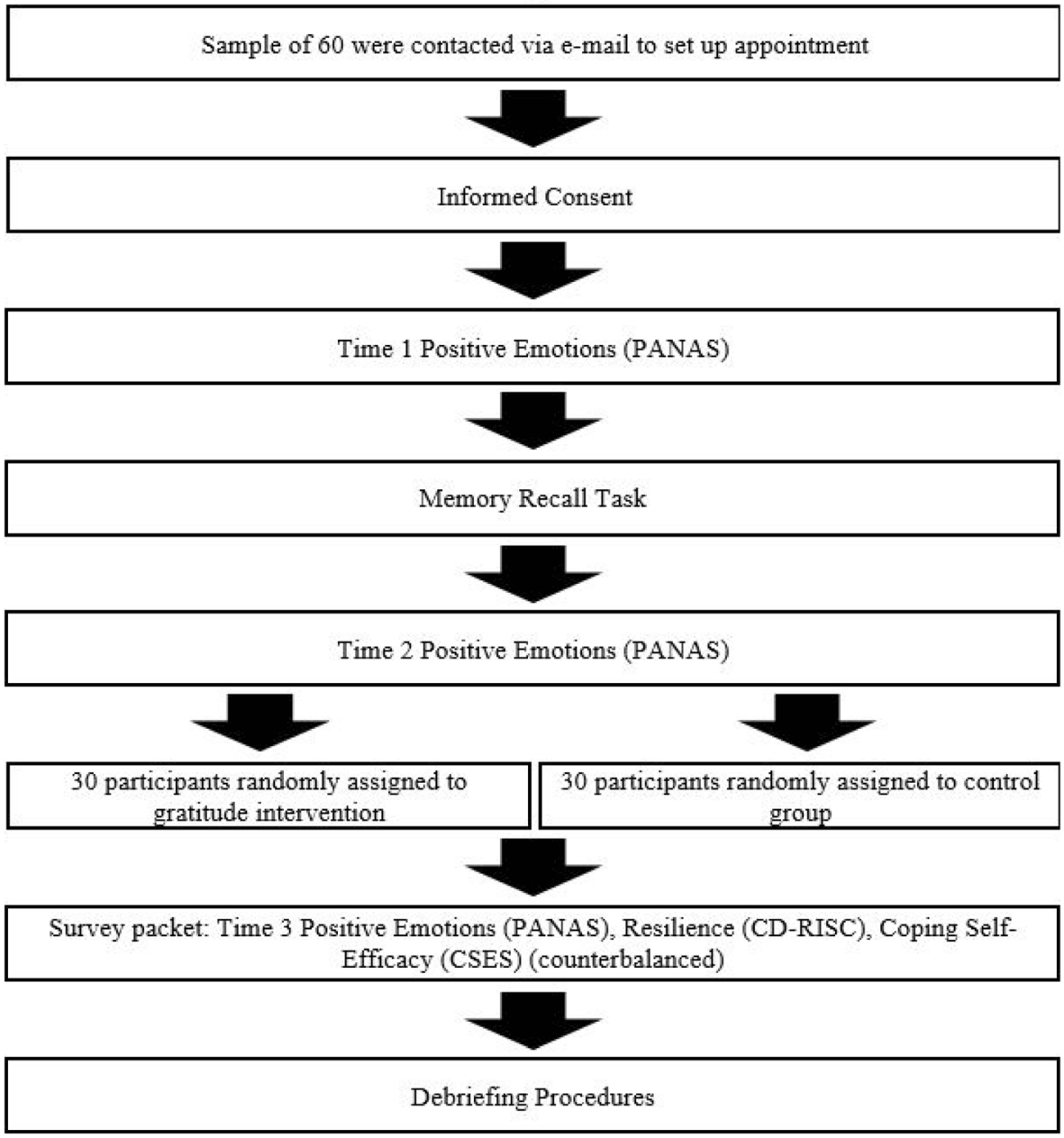

Fig. 1 A flow chart of the study's procedures

of wellbeing scores, which represented the first cycle of the RSS scheme. We repeated those steps 9 times to select 30 participants for each intervention. Once we established two 30-participant groups with comparable wellbeing scores, we randomly assigned each 30-participant group to a condition via a coin toss.

Selected participants were contacted via e-mail to schedule a face-to-face data collection session. Of those 60 participants, 48 attended their scheduled appointment 
and they comprised the final sample (as described in the Participants section). At the beginning of the data collection session participants were asked to read and sign an informed consent form and then complete a baseline measure of positive emotions. Then participants were instructed to complete the 15-min positive experience task (see below). Following completion of the positive experience task, participants completed the measure of positive emotions again. Then, depending on their random assignment, participants either completed the gratitude intervention (see below) or the control task (see below). Afterwards, participants completed a counter-balanced survey packet which included the measure of positive emotions, in addition to measures of resilience and coping self-efficacy. Finally, the participants were debriefed and thanked for their participation. Participants completed all tasks within $60 \mathrm{~min}$. Finally, it is important to note the scoring strategy used in data analysis. Specifically, we used sum scores to generate scale scores. Sum scores are a common method of measuring for perceptions, mood, and attitudes in psychological surveys (Cohen et al. 2013), especially when there is low levels of missing data. In the current study, less than $1 \%$ of the data were reported missing. Thus, sum scores appear to be a viable measurement strategy to analyze our data. However, others suggest average scores offer more methodological benefits when compared to sum scores. To ensure our measurement strategy did not affect key findings, we ran the analyses using both methods. Reported findings were comparable for sum scores vs. average scores.

\subsection{Positive Experience Task}

To induce a positive experience, participants were asked to complete a positive memory recall exercise. The researcher asked participants to recall a significant accomplishment they achieved over the last few years. After participants identified their accomplishment, the researcher assigned a small journaling task. Specifically, participants were asked to write a short, yet detailed story of how they achieved their accomplishment. To increase imageability of the memory recall task (Rasmussen and Berntsen 2014), participants were asked to comment on how important emotions were present during the beginning, middle, and final stages of their accomplishment story. In addition, participants were asked to identify how they overcame barriers to achieve their accomplishment. In total, participants were told to narrate their story using 400 words or more on a notebook computer.

\subsection{Conditions}

\subsubsection{Gratitude Intervention}

Participants randomly assigned to the gratitude intervention were asked to complete a brief listing task. Most gratitude-based exercises include a listing component (Davis et al. 2016). We asked participants to mindfully reflect upon and identify different factors for which they were grateful or thankful. Participants were initially handed a work-sheet to help them list and organize factors for which they were grateful. On the worksheet, participants could list multiple factors under four different categories for which people typically express gratitude: personal strengths, beliefs and attitudes, other people, and other factors. Then participants were given another worksheet to help them estimate the degree to which each of the previously identified factors contributed to their personal success. Participants completed the gratitude intervention in $15 \mathrm{~min}$. 
Control Intervention Participants randomly assigned to the control group were asked to continue to reflect upon and process their positive experience in the lab. The research assistant left the room while the participants processed their experience. The purpose of the control task was to simulate typical cognitive processing without the aid of a structured gratitude-based exercise. In the control condition, participants processed their experience for $15 \mathrm{~min}$.

\subsection{Measures}

Ryff Scales for Psychological Wellbeing-Short Form (RSPWB-SF) The RSPWB-SF measures six key dimensions of wellbeing with 18-items (Ryff 1989; Ryff and Keyes 1995). These dimensions include autonomy, environmental mastery, personal growth, positive relations with others, purpose in life, and self-acceptance. Each item is rated on a 6-point scale from 1 (Disagree Strongly) to 6 (Agree Strongly). Internal consistency coefficients for these dimension scores generally fall below the minimal standard of acceptance $(\alpha>.70)$ and the multidimensional factor structure is generally more tenuous in studies using smaller sample sizes (Kafka and Kozma 2002). For this reason total scores were used for the participant data from this measure. Total scores range from 18 to 108 with higher scores reflecting greater psychological wellbeing. Given consistently high correlations with positive emotions and coping resources (Proctor et al. 2011), RSPWB-SF total scores were used as an auxiliary covariate. In the current study, the RSPWB-SF demonstrated solid internal consistency $(\alpha=.72)$.

RSPWB-SF scores were exclusively used to ensure the effects of wellbeing were equally distributed across all conditions. Participants' scores on the RSPWB-SF ranged from 62 to 108 with a mean of $82.52(S D=9.33)$. When compared against established cut-off scores, our sample's mean wellbeing score is high. We used an independent samples t-test to determine if the wellbeing scores were comparable across groups. Individuals in the gratitude intervention $(M=81.21, S D=9.77)$ reported comparable wellbeing scores compared to those in the control group $(M=83.83, S D=8.82), t(58)=-1.09, p>.05$.

Positive and Negative Affect Scale - Positive Affect (PANAS-PA) The PANAS-PA is a 10item measure of positive feelings resulting from positive engagement with one's environment (Watson et al. 1988). Participants were asked to rate the extent to which they felt interested, excited, strong, enthusiastic, alert, inspired, determined, attentive, and active in the current moment. Participants were asked to rate their emotions on a 100-point scale from 1 (Very Slightly/Not at All) to 100 (Extremely). Total scores were summed and ranged from 10 to 1000 with higher scores reflecting greater positive emotions. The PANAS-PA demonstrates solid internal consistency ( $\alpha=.84$ to .88 ) and convergent validity with measures of positive coping (Burns et al. 2008). In the current study, the PANAS-PA demonstrated excellent internal consistency ( $\alpha=.90$ to .95 ) across different administrations.

We used a 1 to 100 rating scale, which is a deviation from the typical 1 to 5 rating scale, to assess participant reports with the PANAS-PA. Trends in college student responses to positive psychological surveys and best practices in maximizing variability were our considerations in making this decision. College student responses to positive psychological surveys tend to be high, with a number of respondents reporting near-maximum or maximum scores. For instance, $60 \%$ of the current sample scored within the upper $25 \%$ quadrant on the RSPWB-SF. This pattern of responding can indicate the presence of a ceiling effect. Ceiling effects are rating score limitations that 
(a) reduce the true range of scores, (b) underestimate variability, and (c) negatively affect estimates of reliability and validity for a specific measure, leading to biased and inaccurate research findings (Uttl 2005). One method of minimizing the potential for a ceiling effects is to increase the response range by which an individual can estimate their current level of functioning. We choose to utilize a 1 to 100 score range because it is consistent clinical recommendations in estimating mood (e.g., Subjective Units of Distress Scale; Franklin and Foa 2014).

Connor-Davidson Resilience Scale - 10 Item (CD-RISC 10) The CD-RISC 10 item scale is a brief measure of positive adaption in the face of stress and trauma (Connor and Davidson 2003). Items are rated on a 5-point scale from 0 (Not True at All) to 4 (True Nearly all the Time). Total scores range from 0 to 40 with higher scores reflecting higher levels of resilience. The CD-RISC 10 demonstrates internal consistency $(\alpha=.85)$ and convergent validity with measures of psychiatric symptoms (Campbell-Sills and Stein 2007). In the current study, the CD-RISC 10 demonstrated solid internal consistency $(\alpha=.83)$.

Coping Self-Efficacy Scale (CSES) The CSES is a 26-item measure of confidence in one's ability to cope effectively with adversity (Chesney et al. 2006). Items are scored on an 11-point scale from 0 (Cannot do at all) to 10 (Certain I can do). Total scores range from 0 to 260 with higher scores reflecting greater levels of coping self-efficacy. Psychometric evaluation of the CSES reveals excellent internal consistency scores $(\alpha=.95)$ and high convergent validity with measures of problem-focused coping and planful problem solving (Chesney et al. 2006). In the current study, the CSES demonstrated solid internal consistency $(\alpha=.88)$.

\section{Results}

\subsection{Preliminary Analyses}

Manipulation Check The positive experience task was intended to induce positive emotions. To ensure the task elicited the intended effect, we examined within subject

Table 1 Descriptive statistics and intervention differences on the study's main variables

\begin{tabular}{lll}
\hline Variables & \multicolumn{2}{l}{ Interventions } \\
\cline { 2 - 3 } & \multicolumn{1}{l}{ Gratitude Group (M, SD) Control Group (M, SD) } \\
\hline Time 1 Positive Emotions & $441.68(195.76)$ & $436.52(184.92)$ \\
Time 2 Positive Emotions & $556.40(178.18)$ & $574.22(215.08)$ \\
Time 3 Positive Emotions & $561.88(230.92)$ & $400.52(229.54)^{* *}$ \\
Resilience & $31.60(5.45)$ & $28.13(4.58)^{*}$ \\
Coping Self-Efficacy & $193.88(39.83)$ & $165.65(32.08)^{* *}$ \\
\hline
\end{tabular}

Asterisks denote significant differences between participants in the gratitude vs. the control groups $* p<.05, * * p<.01$ 
differences in baseline and post response reports of positive emotions. Mean scores, averaged across participants, for positive emotions are depicted in Table 1. A within subjects t-test confirmed participants reported significantly elevated levels of positive emotions after engaging in the positive experience task, $t(47)=-6.35, p<.01, r=.74$.

Attrition Check Twelve individuals who were contacted did not attend their scheduled testing session. It is important to evaluate whether these 12 individuals were different in any way from the 48 individuals who completed all experimental tasks. Because we screened all participants, we were able to evaluate differences between individuals who failed to attend their schedule session $(n=12)$ and those who completed the experimental tasks $(n=48)$ on wellbeing scores. Results from an independent t-test revealed a non-significant difference on wellbeing, $t(58)=.178, p=n s, r=.02$, suggesting individuals who did not attend were not different from individuals who did attend on levels of wellbeing.

\subsection{Hypothesis 1}

The first hypothesis was tested with a 2 (condition: gratitude or control) $\times 3$ (time: time 1, time 2, time 3) mixed ANOVA to assess for significant differences in positive emotion scores. Our analysis revealed a significant main effect for time, $F(2,45)=12.25, p<.01$, $\eta_{p}{ }^{2}=.21$ and a non-significant main effect for condition, $F(1,46)=12.25, p=n s$, $\eta_{p}{ }^{2}=.02$. Results also revealed a significant condition $\mathrm{x}$ time interaction, $F(2,45)=$ $10.21, p<.01, \eta_{p}{ }^{2}=.18$, which is indicative of a moderate effect (Cohen 1988). To break down the interaction effect, we ran a series of t-tests to determine within and between participant differences on positive emotions. As seen in Fig. 2, self-reports of positive emotions did not differ at Time 1 between participants in the gratitude $(M=441.68$, $S D=195.76)$ and control $(M=436.52, S D=184.91)$ groups, $t(46)=.09, p>.05$. A similar pattern of findings was revealed at Time 2, where participants in the gratitude group $(M=556.40, S D=178.18)$ and control group $(M=574.22, S D=215.08)$ reported comparable scores, $t(46)=-.31, p>.05$. However, between group differences were detected at Time 3. Participants in the gratitude condition reported greater levels of positive emotions $(M=561.88, S D=280.67)$ compared to those in the control condition $(M=400.52, S D=229.54), t(46)=2.49, p<.05$.

With regard to within group differences, individuals in the gratitude intervention reported comparable scores from Time 2 to Time 3, $M_{\text {difference }}=-29.72$, $t(24)=-.71, p>.05$. These patterns of findings suggest individuals assigned to

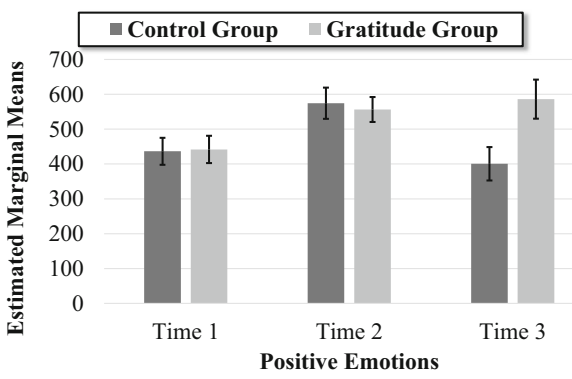

Fig. 2 A bar graph tracking the interaction effects of condition (control vs. gratitude) and time on positive emotions 
the gratitude task were able to sustain (not enhance) higher levels of positive emotions from Time 2 to Time 3. This effect was not evident among individuals assigned to the control group. Reported positive emotions decreased from Time 2 to Time 3 in the control group, $M_{\text {difference }}=173.69, t(22)=6.39, p<.01$. In combination, these findings support our hypothesis that expressing gratitude serves to sustain elevated positive emotional states in the short term.

\subsection{Hypothesis 2}

The second hypothesis was tested with a MANOVA with two dependent variables: resilience and coping self-efficacy. Our analysis revealed a significant multivariate effect, $\lambda=.86, F(2,45)=3.73, p<.01, \eta_{p}^{2}=.14$. As shown in Figs. 3 and 4 , individuals in the gratitude condition reported higher levels of resilience and coping self-efficacy compared to individuals in the control condition. Participants who completed the gratitude intervention $(M=31.60, S D=5.45)$ reported higher levels of resilience compared to the control group $(M=28.13, S D=4.57), F(1,46)=5.65$, $p<.05, \eta_{p}{ }^{2}=.11$. Similar findings were reveled for coping self-efficacy, $F(1,46)=$ $7.32, p<.05, \eta_{p}{ }^{2}=14$, such that participants who completed the gratitude intervention $(M=165.65, S D=39.83)$ scored significantly higher than participants in the control group $(M=139.88, S D=32.07)$. These findings support our hypothesis that gratitude interventions contribute to higher perceptions of coping resources.

\section{Discussion}

The aim of the current study was to examine pathways by which gratitude contributes to wellbeing outcomes (positive emotions and coping resources). Our study was the first to consider the impact of a gratitude intervention on different wellbeing outcomes in the context of a positive experience (i.e., positive memory recall). Statistical analyses revealed (a) a significant interaction of condition and time on positive emotions and (b) a significant main effect of

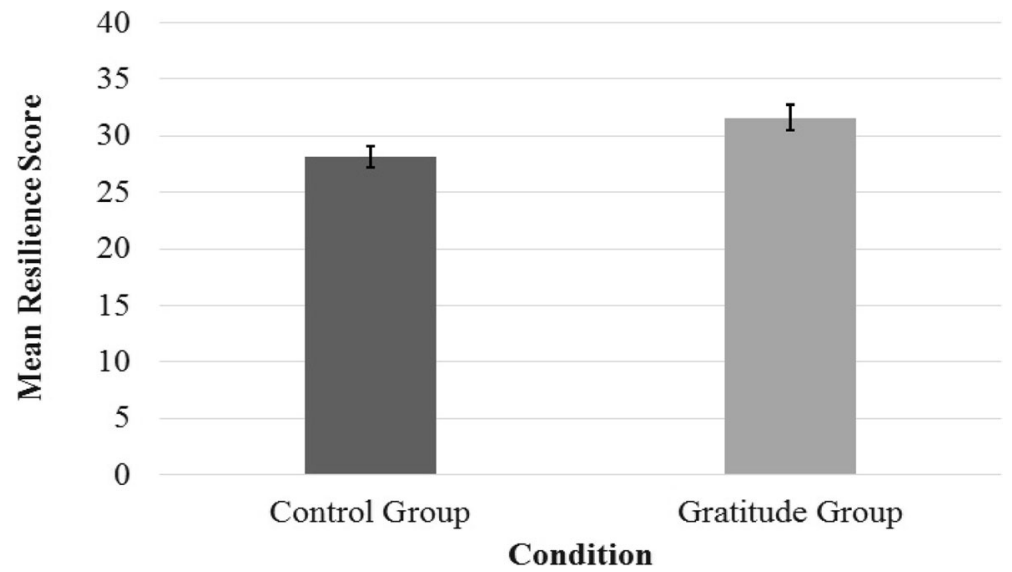

Fig. 3 Bar graph depicting mean scores and standard errors for condition (control vs. gratitude) on resilience 


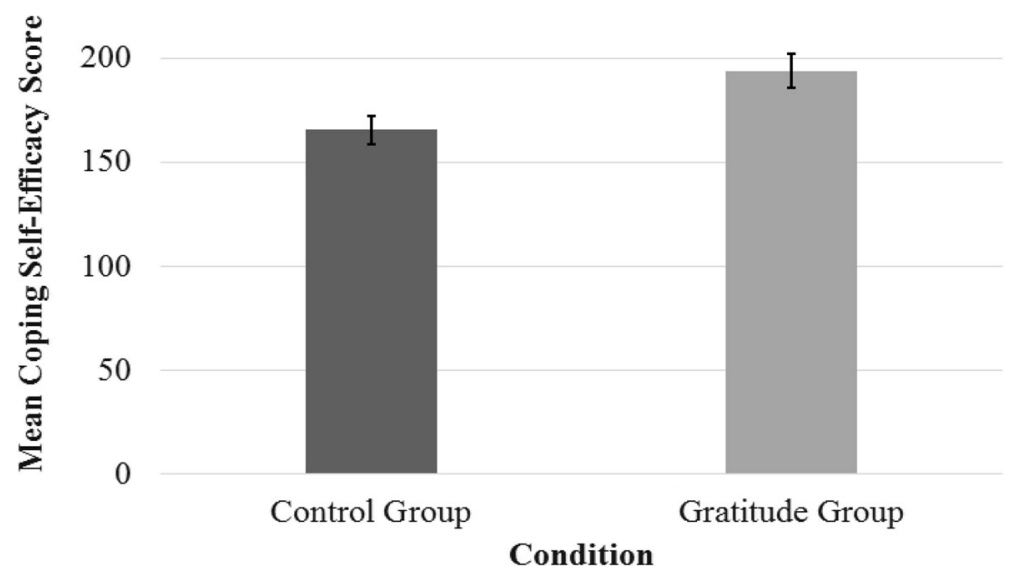

Fig. 4 Bar graph depicting mean scores and standard errors for condition (control vs. gratitude) on coping self-efficacy

condition on self-reported levels of coping resources (resilience and coping selfefficacy). Overall, within the parameters of the current study, these findings provide additional support for gratitude as an effective upregulation strategy.

\subsection{Gratitude and Positive Emotions}

Participants randomly assigned to the gratitude intervention group reported higher levels of positive emotion at Time 3 compared to participants in the control group. This finding is consistent with positive emotion regulation theory (Gross 2015) and highlights the potential for gratitude interventions to upregulate positive emotions. Moreover, the pattern of our findings provide some clarity regarding the extent (enhance vs. maintain) to which gratitude interventions may upregulate positive emotions. Of note, positive emotion scores for individuals assigned to the gratitude intervention did not statistically increase from Time 2 to Time 3 . This finding is not consistent with the position that gratitude interventions enhance positive emotions resulting from a positive experience. Instead, our findings support a steady pattern of elevated positive emotions, which indicates gratitude interventions may help maintain or prolong (in the short term) positive emotions resulting from a positive experience. This pattern was not detected in the control group, where positive emotions scores decreased from Time 2 to Time 3.

This experimental finding is a meaningful addition to the literature, especially considering previous studies have failed to garner clear evidence for a causal link between gratitude interventions and heightened positive emotional states (Kerr et al. 2015). One important element of our study may help clarify the inconsistency between our findings and those of other researchers. Specifically, we examined the impact of a gratitude intervention on positive emotions after a positive experience (i.e., positive memory recall). Given the current findings, we suggest researchers direct more attention to the maintenance features of gratitude interventions. Gratitude interventions may not be powerful enough to induce elevations in positive emotions by themselves. 
However, gratitude interventions may be well-suited to help individuals capitalize on existing positive experiences as a means to maintain elevated levels of positive emotions. This is an important distinction and provides insight on when and how clinicians should employ gratitude interventions.

Despite these promising findings, the current study is preliminary and so future research is needed to further explore this area and answer additional questions about gratitude interventions. For instance, further study is needed to determine if the beneficial effects (positive emotional states) of gratitude interventions endure for long periods of time. Longitudinal designs are often required to generate decisive findings regarding if and how gratitude interventions contribute to enduring positive psychological resources (Wood et al. 2008). It would be ideal to track the effects of gratitude interventions over time to determine if the benefits identified in the current study are sustained in the long term, 6 to 12 months.

In addition, researchers should also examine gratitude as a conditional effect by which positive experiences contribute to positive emotions. Research consistently contends not all reactions to positive events generate positive emotions (Quoidbach et al. 2010). Therefore, identifying variables that facilitate or inhibit the relationship between positive experiences and positive emotions would be a fruitful avenue of investigation. The current findings support the potential for gratitude interventions to maintain a strong connection between positive experiences and elevated levels of positive emotions. However, our design does not allow us to specifically infer moderation at this level. In order to investigate the conditional effects of gratitude, future researchers should experimentally determine if experience (positive, neutral) interacts with intervention (gratitude, control) to explain variation in positive emotion scores. Results from such a study would help determine whether gratitude interventions serve as a promotional factor in helping individuals use positive experiences to increase positive emotions. This may be an especially meaningful finding as select groups of individuals psychologically dampen positive experiences, which inhibits the experience of positive emotions (Quoidbach et al. 2010). In this case, gratitude interventions may be a useful method of helping individuals capitalize on positive experiences in a way that maintains and extends long-term positive emotional wellbeing. Overall, employing longitudinal and moderation-based designs may provide further clarification in determining if gratitude interventions can maintain and extend positive emotions generated from a positive experience.

\subsection{Gratitude and Coping Resources}

Consistent with expectation, the analyses also revealed a significant main effect of condition on levels of resilience and coping self-efficacy. Specifically, participants randomly assigned to the gratitude intervention reported higher levels of resilience and coping self-efficacy compared to the control group. By requiring all participants to complete a positive experience task, we were able to induce comparable levels of positive emotions in participants before random assignment into different conditions. Because levels of positive emotions were comparable for intervention and control participants, the significant differences in resilience and coping self-efficacy are likely due to the effects of the gratitude intervention, and not positive emotions resulting from the positive experience task. 
Overall, these findings provide some support for the build hypothesis of the broaden-and- build theory (Fredrickson 2004), particularly gratitude interventions employed after a positive experience build important personal resources. Importantly, our results extend the literature by specifying what types of resources gratitude helps build. Most studies demonstrate the beneficial effects of gratitude on social resource development (e.g., strengthening existing support systems, forging new relationships; Kerr et al. 2015). Our study was the first to experimentally examine the causal link between gratitude and coping resources. Specifically, our findings highlight the potential for gratitude interventions to increase perceptions of resilience and efficacy in managing stressful circumstances.

Further research is needed to validate these results and examine how gratitude promotes greater coping resources. For instance, it may be beneficial to examine gratitude in the context of stress induction tasks to determine the effectiveness of different interventions in terms of increasing positive coping perceptions and outcomes. In addition, it is important to determine if gratitude interventions uniquely contribute to coping resource development. Because gratitude is classified as a specific feature of positive affect, it is important to distinguish if and when gratitude can promote better coping outcomes above and beyond the effects of generalized positive affect (Watkins 2004). In our study, we examined the effects of gratitude on different coping resources after a positive experience (which resulted in elevated levels of positive emotions). However, since we did not manipulate who received the positive experience task, we were unable to control for the effects of generalized positive affect. In the future, it will be important to manipulate positive experiences to determine if gratitude interventions can explain elevations in coping resource scores above and beyond the impact of generalized positive affect. Finally, it is important to determine if elevations in coping resources are due to the effects of specific gratitude interventions. In the current study, we employed the most common gratitude intervention, a listing task. However, gratitude interventions include other categories (e.g., contemplation, behavioral expression; Wood et al. 2010). Given the diversity of gratitude interventions, future research should comparatively examine the causal effects of different tasks on resilience and coping self-efficacy outcomes.

Despite the need for further investigation, our findings offer preliminary support for the impact of gratitude on coping resource development. If our findings are supported by future research, gratitude may be well suited as a primary prevention strategy to help individuals build resources needed to navigate stressful circumstances.

\subsection{Practical Implications}

Clinical and counseling researchers aim to identify pathways by which individuals can bolster wellbeing and increase overall quality of life. Given the current literature, gratitude appears to be a promising pathway for activating different wellbeing outcomes. However, there is some confusion regarding how to maximize the effects of gratitude interventions on wellbeing outcomes. In this vein, our findings offer some unique insights regarding the administration of gratitude interventions. Specifically, mental health service providers may need to think carefully about the context in which gratitude interventions are administered. Instead of promoting isolated expressions of gratitude, it may be more beneficial to teach individuals how to induce gratitude after a positive experience. In this way, gratitude interventions may help individuals maintain positive 
emotional expressions (e.g., joy, pleasure, contentment) and build coping resources. Overall, our findings support gratitude interventions as psychologically beneficial. However, emotional and coping outcomes may be strengthened by gratitude interventions when they are administered after a positive experience.

\subsection{Limitations}

Several limitations to the current study need to be acknowledged. First, our findings may have limited generalizability due to the composition of our sample. Specifically, the majority of our sample was comprised of young women with above average wellbeing scores. Given the relatively homogenous nature of our sample, it is unknown whether our findings will generalize to clinical, older adult, or predominantly male samples. It is particularly important that future research examine the effects of gratitude interventions on samples reporting clinical levels of depressive and anxious symptoms. Researchers assert a strong need to develop positive psychological interventions that help individuals in distress cultivate and extend positive emotions and coping resources (Horner et al. 2014; Waugh and Koster 2015). Second, social and emotional capital gained from positive psychological interventions increases with age (Sin and Lyubomirsky 2009). Considering this trend, it is important to re-evaluate our findings with a sample of older adults. It is quite possible that the use of a younger sample may have under-estimated the effects of our gratitude intervention.

Third, our study employed an unstructured, processing-based control task. Although our control task may contrast well against a structured gratitude intervention, it does not actively instruct individuals to process their experience in a generalized or standard manner. As such, future research is needed to examine gratitude interventions against unstructured and structured control tasks. For instance, one could devise a more structured control task that asks participants to think about themselves in a specific setting or situation. Comparing our gratitude intervention against a diverse range of control tasks may increase the confidence in our identified effects. In addition, we could contrast our gratitude intervention against other established upregulation strategies (e.g., visualizing best possible selves, intensifying the moment; Bryant and Veroff 2007; Sheldon and Lyubomirsky 2006). Such comparisons may provide incremental validity in designating gratitude interventions as an effective strategy to upregulate positive emotions and coping resources. Fourth, the study did not measure for change in resilience and coping self-efficacy scores. This limits interpretations regarding whether short-term gratitude interventions can increase important coping resources. Future studies may want to evaluate within-participant differences in coping resources by gratitude intervention (gratitude vs. control) using pre-test, post-test designs. Such studies would offer more concrete evidence for gratitude as a causal agent in terms of increasing coping resources.

Finally, we relied heavily on self-report data, which is susceptible to increased error via social demand characteristics and method variance. To combat these confounds, researchers may need to examine the effects of our intervention using more behavioral, observational, and physiological measures of positive emotions, resilience, and coping self-efficacy. For instance, resilience is often measured by a number of cardiovascular stress response assessments (Howard and Hughes 2012), including finger pulse amplitude, galvanic skin temperature, pulse transmission time, diastolic blood pressure, 
among others. In this vein, we recommend future researchers examine the effects of gratitude interventions using more robust measures of wellbeing outcomes. For example, assessing resilience through cardiovascular responses to environmentally challenging situations may provide a richer estimate of the degree to which gratitude interventions increase coping resources.

\subsection{Conclusions}

Our findings provide a significant extension of the gratitude intervention literature. Researchers call for creative pathways by which gratitude exerts its positive influence on different wellbeing outcomes (Davis et al. 2016). Our study answers this call in two ways. First, a gratitude intervention was found to be well suited to capitalize on positive experiences to maintain elevated levels of positive emotions in the short term. Second, our study is one of the first to draw a direct causal connection between gratitude and different coping resources, namely, resilience and coping self-efficacy. The current study offers some important insights on how mental health clinicians can effectively employ gratitude interventions. Of note, clinicians should consider using gratitude exercises after a positive experience to maintain positive emotions and build important coping resources.

\section{Compliance with Ethical Standards}

Conflict of Interest On behalf of all authors, the corresponding author states that there is no conflict of interest.

Ethical Approval All procedures performed in studies involving human participants were in accordance with the ethical standards of the institutional and/or national research committee and with the 1964 Helsinki declaration and its later amendments or comparable ethical standards.

The data collection procedures were approved by Georgia Southern University's IRB Board. All data were collected in a manner consistent with the approved IRB application.

Informed Consent Informed consent was obtained from all individual participants included in the study.

\section{References}

Bryant, F. B. (2003). Savoring beliefs inventory (SBI): A scale for measuring beliefs about savouring. Journal of Mental Health, 12(2), 175-196. https://doi.org/10.1080/0963823031000103489.

Bryant, F. B., \& Veroff, J. (2007). Savoring: A new model of positive experience. Mahwah: Lawrence Erlbaum Associates Publishers.

Burns, A. B., Brown, J. S., Sachs-Ericsson, N., Plant, E. A., Curtis, J. T., Fredrickson, B. L., \& Joiner, T. E. (2008). Upward spirals of positive emotion and coping: Replication, extension, and initial exploration of neurochemical substrates. Personality and Individual Differences, 44(2), 360-370. https://doi.org/10.1016/j.paid.2007.08.015.

Campbell-Sills, L., \& Stein, M. B. (2007). Psychometric analysis and refinement of the Connor-Davidson resilience scale (CD-RISC): Validation of a 10-item measure of resilience. Journal of Traumatic Stress, 20(6), 1019-1028. https://doi.org/10.1002/jts.20271. 
Chesney, M. A., Neilands, T. B., Chambers, D. B., Taylor, J. M., \& Folkman, S. (2006). A validity and reliability study of the coping self-efficacy scale. British Journal of Health Psychology, 11(3), 421-437. https://doi.org/10.1348/135910705X53155.

Cohen, J. (1988). Statistical power analysis for the behavioral sciences (2nd ed.). Hillsdale: Erlbaum.

Cohen, R. J., Swerdlik, M. E., \& Sturman, E. D. (2013). Psychological testing and assessment: An introduction to tests and measurement (8th edn.). New York: McGraw-Hill.

Cohn, M. A., Fredrickson, B. L., Brown, S. L., Mikels, J. A., \& Conway, A. M. (2009). Happiness unpacked: Positive emotions increase life satisfaction by building resilience. Emotion, 9(3), 361-368. https://doi.org/10.1037/a0015952.

Connor, K. M., \& Davidson, J. R. (2003). Development of a new resilience scale: The Connor-Davidson resilience scale (CD-RISC). Depression and Anxiety, 18(2), 76-82. https://doi.org/10.1002/da.10113.

Davis, D. E., Choe, E., Meyers, J., Wade, N., Varjas, K., Gifford, A., et al. (2016). Thankful for the little things: A meta-analysis of gratitude interventions. Journal of Counseling Psychology, 63(1), 20-31. https://doi.org/10.1037/cou0000107.

Donner, A., \& Zou, G. Y. (2007). Methods for the statistical analysis of binary data in split-mouth designs with baseline measurements. Statistics in Medicine, 26(18), 3476-3486.

Egger, J., Graham, P. J., Carter, C. M., Gumley, D., \& Soothill, J. F. (1985). Controlled trial of oligoantigenic treatment in the hyperkinetic syndrome. Lancet, 9(1), 540-545.

Emmons, R. A., \& McCullough, M. E. (2003). Counting blessings versus burdens: An experimental investigation of gratitude and subjective well-being in daily life. Journal of Personality and Social Psychology, 84(2), 377-389. https://doi.org/10.1037/0022-3514.84.2.377.

Franklin, M. E., \& Foa, E. B. (2014). Obsessive-compulsive disorder. In D. H. Barlow \& D. H. Barlow (Eds.), Clinical handbook of psychological disorders: A step-by-step treatment manual (pp. 155-205). New York: Guilford Press.

Fredrickson, B. L. (2001). The role of positive emotions in positive psychology: The broaden-andbuild theory of positive emotions. American Psychologist, 56(3), 218-226. https://doi. org/10.1037/0003-066X.56.3.218.

Fredrickson, B. L. (2004). Gratitude, like other positive emotions, broadens and builds. In R. A. Emmons, M. E. McCullough, R. A. Emmons, \& M. E. McCullough (Eds.), The psychology of gratitude (pp. 145-166). New York: Oxford University Press. https://doi.org/10.1093/acprof:oso/9780195150100.003.0008.

Fredrickson, B. L., Cohn, M. A., Coffey, K. A., Pek, J., \& Finkel, S. M. (2008). Open hearts build lives: Positive emotions, induced through loving-kindness meditation, build consequential personal resources. Journal of Personality and Social Psychology, 95(5), 1045-1062. https://doi.org/10.1037/a0013262.

Gross, J. J. (1998). The emerging field of emotion regulation: An integrative review. Review of General Psychology, 2(3), 271-299. https://doi.org/10.1037/1089-2680.2.3.271.

Gross, J. J. (2015). Emotion regulation: Current status and future prospects. Psychological Inquiry, 26(1), 126. https://doi.org/10.1080/1047840X.2014.940781.

Gross, J. J., \& Muñoz, R. F. (1995). Emotion regulation and mental health. Clinical Psychology: Science and Practice, 2(2), 151-164. https://doi.org/10.1111/j.1468-2850.1995.tb00036.x.

Horner, M. S., Siegle, G. J., Schwartz, R. M., Price, R. B., Haggerty, A. E., Collier, A., \& Friedman, E. S. (2014). C'mon get happy: Reduced magnitude and duration of response during a positive-affect induction in depression. Depression and Anxiety, 31(11), 952-960. https://doi.org/10.1002/da.22244.

Howard, S., \& Hughes, B. M. (2012). Benefit of social support for resilience-building is contingent on social context: Examining cardiovascular adaptation to recurrent stress in women. Anxiety, Stress \& Coping: An International Journal, 25(4), 411-423. https://doi.org/10.1080/10615806.2011.640933.

Isen, A. M. (1999). Positive affect. In T. Dalgleish, M. J. Power, T. Dalgleish, \& M. J. Power (Eds.), Handbook of cognition and emotion (pp. 521-539). New York: Wiley. https://doi. org/10.1002/0470013494.ch25.

Jose, P. E., Lim, B. T., \& Bryant, F. B. (2012). Does savoring increase happiness? A daily diary study. The Journal of Positive Psychology, 7(3), 176-187. https://doi.org/10.1080/17439760.2012.671345.

Kafka, G. J., \& Kozma, A. (2002). The construct validity of Ryff's Scales of Psychological Well-Being (SPWB) and their relationship to measures of subjective well-being. Social Indicators Research, 57(2), 171-190. https://doi.org/10.1023/A:1014451725204.

Kazdin, A. E. (2016). Research design in clinical psychology (5th ed.). Boston: PearsonEducation Inc.

Kerr, S. L., O’Donovan, A., \& Pepping, C. A. (2015). Can gratitude and kindness interventions enhance well-being in a clinical sample? Journal of Happiness Studies, 16(1), 17-36. https://doi.org/10.1007/s10902-013-9492-1. 
Layous, K., Lee, H., Choi, I., \& Lyubomirsky, S. (2013). Culture matters when designing a successful happiness-increasing activity: A comparison of the United States and South Korea. Journal of CrossCultural Psychology, 44(8), 1294-1303. https://doi.org/10.1177/0022022113487591.

McIntyre, G. A. (1952). A method for unbiased selective sampling using ranked sets. Australian Journal of Agricultural Research, 3, 385-390. https://doi.org/10.1071/ar9520385.

O' Leary, K., \& Dockray, S. (2015). The effects of two novel gratitude and mindfulness interventions on well-being. The Journal of Alternative and Complementary Medicine, 21(4), 243-245. https://doi.org/10.1089/acm.2014.0119.

Proctor, C., Maltby, J., \& Linley, P. A. (2011). Strengths use as a predictor of well-being and health-related quality of life. Journal of Happiness Studies, 12(1), 153-169. https://doi.org/10.1007/s10902-009-9181-2.

Quoidbach, J., Berry, E. V., Hansenne, M., \& Mikolajczak, M. (2010). Positive emotion regulation and wellbeing: Comparing the impact of eight savoring and dampening strategies. Personality and Individual Differences, 49(5), 368-373. https://doi.org/10.1016/j.paid.2010.03.048.

Quoidbach, J., Mikolajczak, M., \& Gross, J. J. (2015). Positive interventions: An emotion regulation perspective. Psychological Bulletin, 141(3), 655-693. https://doi.org/10.1037/a0038648.

Rash, J. A., Matsuba, M. K., \& Prkachin, K. M. (2011). Gratitude and well-being: Who benefits the most from a gratitude intervention? Applied Psychology. Health and Well-Being, 3(3), 350-369. https://doi. org/10.1111/j.1758-0854.2011.01058.x.

Rasmussen, K. W., \& Berntsen, D. (2014). 'I can see clearly now': The effect of cue imageability on mental time travel. Memory \& Cognition, 42(7), 1063-1075. https://doi.org/10.3758/s13421-014-0414-1.

Rosenberg, A. R., Yi-Frazier, J. P., Wharton, C., Gordon, K., \& Jones, B. (2014). Contributors and inhibitors of resilience among adolescents and young adults with cancer. Journal of Adolescent and Young Adult Oncology, 3(4), 185-193. https://doi.org/10.1089/jayao.2014.0033.

Ruini, C., \& Vescovelli, F. (2013). The role of gratitude in breast cancer: Its relationships with post-traumatic growth, psychological well-being and distress. Journal of Happiness Studies, 14(1), 263-274. https://doi. org/10.1007/s10902-012-9330-x.

Ryff, C. D. (1989). Happiness is everything, or is it? Explorations on the meaning of psychological well-being. Journal of Personality and Social Psychology, 57(6), 1069-1081. https://doi.org/10.1037/0022-3514.57.6.1069.

Ryff, C. D., \& Keyes, C. M. (1995). The structure of psychological well-being revisited. Journal of Personality and Social Psychology, 69(4), 719-727. https://doi.org/10.1037/0022-3514.69.4.719.

Samawi, H. M., \& Abu-Dayyeh, W. (2002). On regression analysis with random regressors using ranked samples. International Journal of Information and Management Sciences, 13, 19-36.

Seligman, M. P. (2011). Flourish. New York: Free Press.

Seligman, M. P., Steen, T. A., Park, N., \& Peterson, C. (2005). Positive psychology Progress: Empirical validation of interventions. American Psychologist, 60(5), 410-421. https://doi.org/10.1037/0003-066X.60.5.410.

Sheldon, K. M., \& Lyubomirsky, S. (2006). How to increase and sustain positive emotion: The effects of expressing gratitude and visualizing best possible selves. The Journal of Positive Psychology, 1(2), 7382. https://doi.org/10.1080/17439760500510676.

Sin, N. L., \& Lyubomirsky, S. (2009). Enhancing well-being and alleviating depressive symptoms with positive psychology interventions: A practice-friendly meta-analysis. Journal of Clinical Psychology, 65(5), 467-487. https://doi.org/10.1002/jclp.20593.

Tugade, M. M., Devlin, H. C., \& Fredrickson, B. L. (2014). Infusing positive emotions into life: The broaden-andbuild theory and a dual-process model of resilience. In M. M. Tugade, M. N. Shiota, L. D. Kirby, M. M. Tugade, M. N. Shiota, \& L. D. Kirby (Eds.), Handbook of positive emotions (pp. 28-43). New York: Guilford Press.

Uttl, B. (2005). Measurement of individual differences: Lessons from memory assessment in research and clinical practice. Psychological Science, 16(6), 460-467. https://doi.org/10.1111/j.0956-7976.2005.01557.x.

Vernon, L. L., Dillon, J. M., \& Steiner, A. W. (2009). Proactive coping, gratitude, and posttraumatic stress disorder in college women. Anxiety, Stress \& Coping: An International Journal, 22(1), 117-127. https://doi.org/10.1080/10615800802203751.

Watkins, P. C. (2004). Gratitude and subjective well-being. In R. A. Emmons, M. E. McCullough, R. A. Emmons, \& M. E. McCullough (Eds.), The psychology of gratitude (pp. 167-192). New York: Oxford University Press. https://doi.org/10.1093/acprof:oso/9780195150100.003.0009.

Watson, D., Clark, L. A., \& Tellegen, A. (1988). Development and validation of brief measures of positive and negative affect: The PANAS scales. Journal of Personality and Social Psychology, 54(6), 1063-1070. https://doi.org/10.1037/0022-3514.54.6.1063.

Waugh, C. E., \& Koster, E. W. (2015). A resilience framework for promoting stable remission from depression. Clinical Psychology Review, 4149-4160. https://doi.org/10.1016/j.cpr.2014.05.004.

Wood, A. M., \& Tarrier, N. (2010). Positive clinical psychology: A new vision and strategy for integrated research and practice. Clinical Psychology Review, 30(7), 819-829. https://doi.org/10.1016/j.cpr.2010.06.003. 
Wood, A. M., Joseph, S., \& Linley, P. A. (2007). Coping style as a psychological resource of grateful people. Journal of Social and Clinical Psychology, 26(9), 1076-1093. https://doi.org/10.1521/jscp.2007.26.9.1076.

Wood, A. M., Maltby, J., Gillett, R., Linley, P. A., \& Joseph, S. (2008). The role of gratitude in the development of social support, stress, and depression: Two longitudinal studies. Journal of Research in Personality, 42(4), 854-871. https://doi.org/10.1016/j.jrp.2007.11.003.

Wood, A. M., Froh, J. J., \& Geraghty, A. A. (2010). Gratitude and well-being: A review and theoretical integration. Clinical Psychology Review, 30(7), 890-905. https://doi.org/10.1016/j.cpr.2010.03.005.

Publisher's Note Springer Nature remains neutral with regard to jurisdictional claims in published maps and institutional affiliations. 